\title{
Moving objects detection algorithm of improved GMM based on 2-D entropy
}

\author{
Gang Feng \\ College of computer, Huanan Normal University Guangzhou 510631 China \\ E-mail: newadat@qq.com
}

Keywords: moving object detection; GMM; 2-D entropy; learning rate

Abstract. Aiming at the background updating slow in Gaussian mixture model in abrupt illumination, 2-D entropy is used to judge the mutation of light, thus changing the learning rate so as to speed up the background modeling. The experimental results show that the method can make the background more accurate in complex scenes, and improve the accuracy of target detection.

\section{Introduction}

Moving object detection is one of the key technologies in computer machine vision, has important application in monitoring, vivo detection and accurate guidance etc [1]. The goal of moving object detection is to distinguish the moving regions from the background, which requires reliable and effective moving object detection. In moving object detection, background is changing, so it is very difficult to locate the foreground object, especially in the scene of video noise, target occlusion, illumination changes and other scenes [2].

In recent years, the methods of extracting moving object from video are temporal differencing method [3], optical flow method [4], background subtraction method [5] and image segmentation method [6]. The Gaussian mixture model (GMM) [5] is the best for moving target detection in the background modeling methods. In order to avoid the model's practical disadvantages, many scholars have proposed different improvements in response to the shortcomings of the GMM. For example, Zivkovic et al. [7] produced a recursive algorithm that estimates the parameters of the mixture and that simultaneously selects the number of components. Kaewtrakulpong et al. [8] amended the GMM parameter updating equation. Li Sun et.al [9] proposed another approach for displaying background in complex scenes. Haiying et.al [10] proposed an altered Gaussian blend background demonstrate in view of the spatial-transient dispersion which utilizes time and space appropriation data.

In order to solve traditional GMMs cannot deal with complex backgrounds and are sensitive to sudden movements of the target and light changes, Liu et al [11] introduce image entropy in GMM. But this method ignores the local illumination mutation, and generally treats the local illumination change as the global illumination change. In addition, the method calculates the one-dimensional entropy of the image to determine the change of illumination, and the one-dimensional entropy of the image ignores the spatial information distribution of the gray level of the image, and both of the two factors lead to the reduction of the detection accuracy.

We propose an algorithm to improve GMM based on 2-D entropy in this paper. The 2-D entropy of image combines the gray value of the pixel neighborhood and the gray value of the current pixel together to form a two element combination, which reflects the spatial characteristics of the gray value, and the two-dimensional entropy of the image describes the aggregation characteristics and spatial characteristics of the gray distribution of the image.

The rest of this paper is organized as follows. Sect. 2 discusses details of the proposed modified GMM based on 2-D entropy; Sect.3 places our experimental results, and the conclusion and future work are summarized in Sect. 4. 


\section{An Improved GMM}

The improved Gaussian mixture background model based on 2-D entropy is a method which uses 2-D entropy judging the light mutation. There are two key ideas, including the threshold basing on the difference between neighbor frame of video and the learning rate when light abrupt changes.

\section{2-D entropy of image}

In a gray image, let the gray level be divided into $m$ values and the average gray level is also divided into the same $m$ values. At each pixel, the average gray-level value of the neighborhood is calculated. This forms a pair: the pixel gray level and the average of the neighborhood. Each pair belongs to a 2 dimensional bin. The total number of bins is obviously $m^{*} m$ and the total number of pixels to be tested is $N^{*} N$. The total number of occurrence $f(i, j)$, of a pair $(i, j)$ divided by the total number of pixels, $N^{*} N$, defines the joint probability mass function, $p_{i j}$ : viz., $p_{i j}=f(i, j) / N^{*} N, i$ and $j=0, \ldots, m-1$. It reflects the gray scale distribution of a pixel position and its surrounding pixel gray distribution characteristics. In our paper, $\mathrm{m}=256$, the 2 -D entropy of image is defined as

$$
H=-\sum_{i=0}^{255} \sum_{j=0}^{255} p_{i j} \ln p_{i j}
$$

\section{Algorithm steps}

According to the theory of entropy, the entropy value of the dark image is smaller, and the brighter image has a larger entropy value. In this paper, we calculate the image 2-D entropy to judge whether the illumination changes. The improved algorithm steps are as follows:

(1) The pre $t$ frames in the video are selected as the training set (the remaining frames are used as the test set). We use the original Gauss mixture model algorithm to model the background region, and the foreground and background are detected in the training set. The foreground area is recorded as $\mathrm{A}_{\mathrm{f}}$, and the background area is denoted as $\mathrm{A}_{\mathrm{b}}$.

(2) With frame $t$, we calculate the difference of 2-D entropy between frame $t$ and frame $t-1$ in $A_{f}$. When the value is greater than $T_{c}$, we think the light mutation is occurs, and denote the $A_{f}$ as $A_{c}$, otherwise as $\mathrm{A}_{\mathrm{s}}$.

$$
\left\{\begin{array}{l}
H\left(X_{t-1}\right)=-\sum_{i=0}^{255} \sum_{j=0}^{255} p_{i j}^{t-1} \ln p_{i j}^{t-1} \\
H\left(X_{t}\right)=-\sum_{i=0}^{255} \sum_{j=0}^{255} p_{i j}^{t} \ln p_{i j}^{t} \\
\left|H\left(X_{t}\right)-H\left(X_{t-1}\right)\right| \geq T_{c}, X_{t} \in A_{c} \\
\left|H\left(X_{t}\right)-H\left(X_{t-1}\right)\right|<T_{c}, X_{t} \in A_{s}
\end{array}\right.
$$

where $H\left(X_{t}\right)$ represents the 2-D entropy of the current frame $\mathrm{t}$, and $H\left(X_{t-1}\right)$ represents the 2-D entropy of the previous frame $\mathrm{t}-1, \mathrm{~T}_{\mathrm{c}}$ is the threshold and $\mathrm{T}_{\mathrm{c}}=0.05$.

(3) In this algorithm, the learning rate of the background region is given a fixed learning rate, and the learning rate of the foreground region is obtained by the following expression:

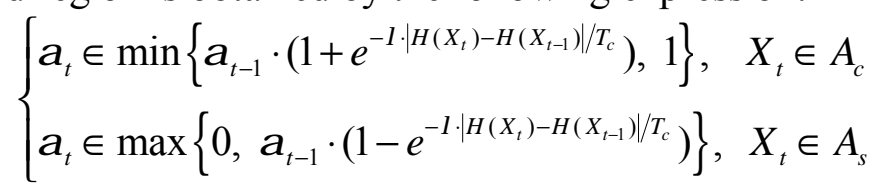

where the learning rate of the current frame is $\alpha_{t}, \lambda$ is the control parameter. 
The flow chart is shown in Figure 1.

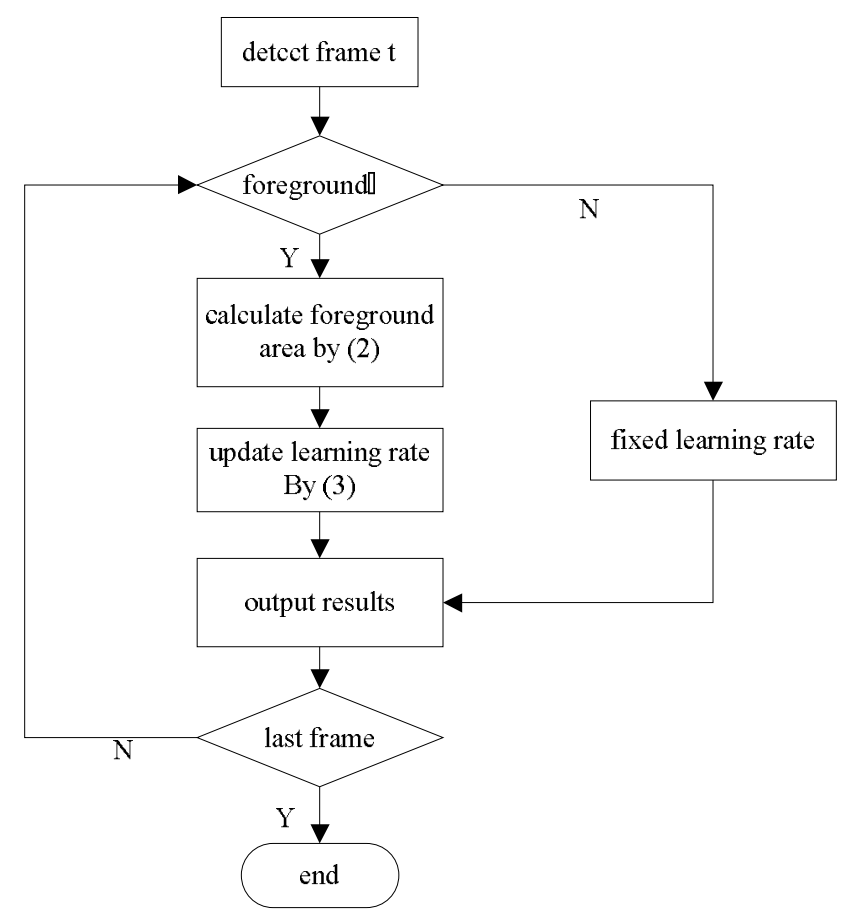

Fig. 1 Improved Gauss mixture background modeling method flow chart

\section{Experimental Results}

The proposed background subtraction algorithms are experimentally validated with a variety of image sequences (Camoutflage, LightSwitch, WavingTrees), Source of video sequence:

http://research.microsoft.com/en-us/um/people/jckrumm/WallFlower/TestImages.htm. The three groups of videos appeared object movement, background light change, tree sway and other scenes. The computer configuration of the experiment: $2.00 \mathrm{GHz}$ (TM) 2 i5 CPU, 4GB RAM. In the algorithm, the number of Gauss is $D=5$, the initial learning rate is $\alpha=0.005$, the threshold is $\tau=0.7$.

The detection results are shown in Fig.2.

In Camoutflage, the background image is a computer display, and the brightness of the computer is constantly changing. We select the first 200 frames as the training set, and the last 152 frames are selected as the test set. Fig.2(a) is the detection results of the 251 st frames, we can see that the Gauss mixture model detects some of the computer monitors as foreground, and there is some empty holes, and our improved method can accurately detect the foreground part.

In LightSwitch, the light dimmed from light. We select the first 500 frames as the training set, and the last 500 frames are slected as the test set. Fig.2(b) is the detection results of the 806th frames, we can see that the Gauss background mixture model detects some shadows, and our improved algorithm can accurately detect the foreground part.

In WavingTrees, the background is the waving branches. We select the first 200 frames as the training set, and the last 185 frames are selected as a test set. Fig.2(c) is the detection of the 325th frames. We can see that the Gauss mixture background model detect some shadows around the man, and our improved algorithm can detect the foreground target more complete.

Through the three scenarios, we can see that the detection method proposed in this paper can detect the foreground object more complete, and there is no large range of holes, and the anti-interference ability is stronger. 


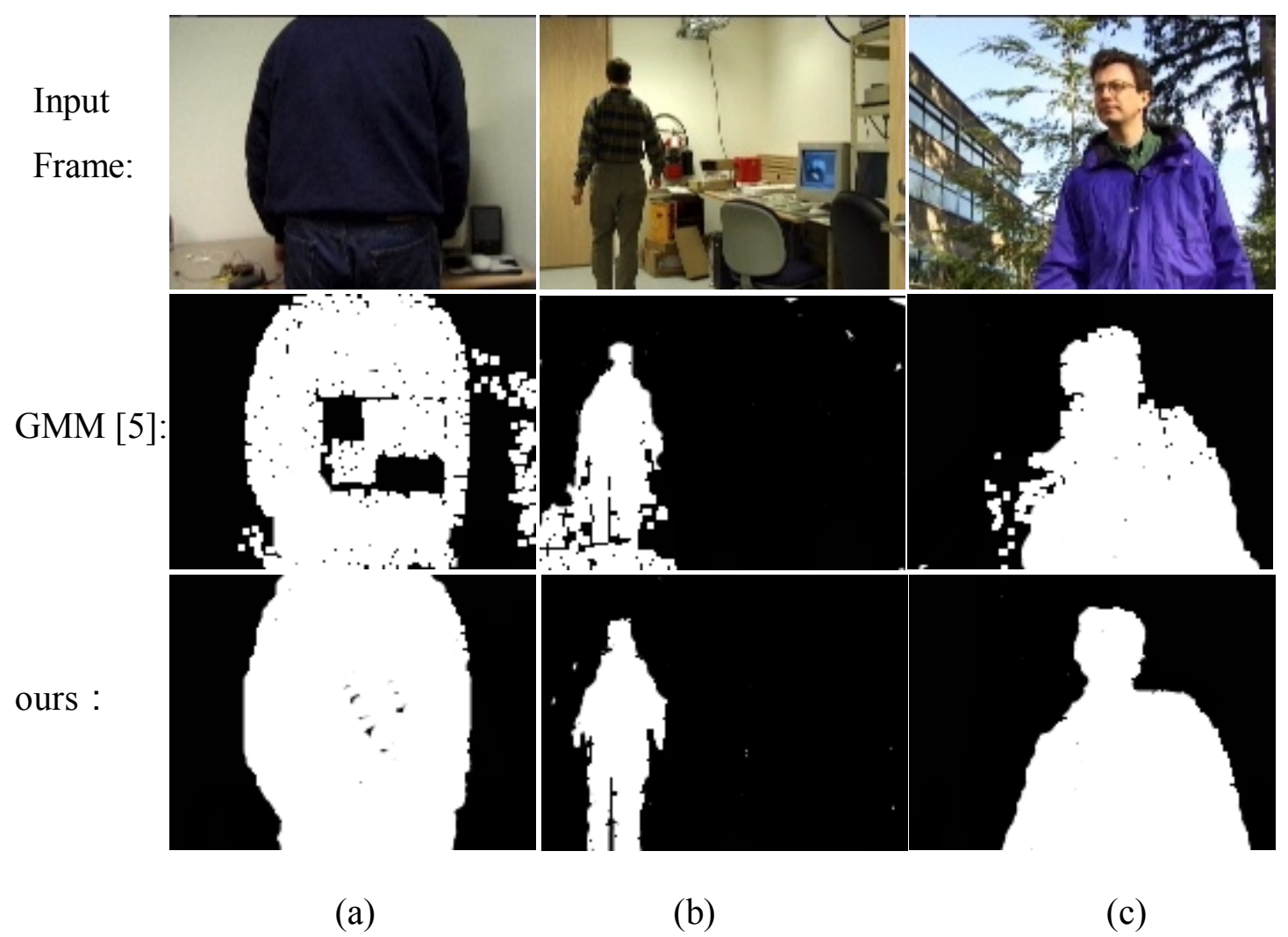

Fig.2 background subtraction results

\section{Conclusions}

Background subtraction is a fundamental research problem in computer vision. According to the traditional Gauss mixture background modeling deficiencies, we propose an improved algorithm, combined with image entropy to adjust the learning rate of the Gauss mixture background model, to adapt to sudden illumination changes. The experimental results show that the improved method is superior to the original Gauss mixture background modeling method.

\section{References}

[1] Alper Yilmaz, Omar Javed and Mubarak Shah. Object Tracking: A Survey, ACM Computing Surveys, Vol. 38, No. 4, Article 13, December 2006.

[2] A.Ramya and Dr. P.Raviraj. A Survey and Comparative Analysis of Moving Object Detection and Tracking, International Journal of Engineering Research \& Technology, Vol. 2 Issue 10, October 2013.

[3] A. J. Lipton, H. Fujiyoshi, and R.S. Patil. Moving target classification and tracking from real-time video, In Applications of Computer Vision, 1998. WACV'98. Proceedings, Fourth IEEE Workshop on, pages 8-14. IEEE, 1998.

[4] J.Barron, D.Fleet and S.Beauchemin. Performance of optical flow Techniques, International Journal of Computer Vision, 1994.

[5] Stauffer C, Grimson W E L. Adaptive background mixture models for real-time tracking[C], Proc of Conference on Computer Vision and Pattern Recognition. pp.246-252, 1999: 
[6] J Shi and J Malik. Normalized cut and image segmentation, IEEE Transactions on Pattern Analysis and Machine Intelligence, vol.22, NO. 8, 2000.

[7] Zivkovic, Z., van der Heijden, F. Recursive unsupervised learning of finite mixture models. IEEE Trans. Pattern Anal. Mach. Intell. 26(5), pp.651-656, 2004

[8] Techawatcharapaikul, C, Kaewtrakulpong, P, Siddhichai, S. Outdoor vehicle and shadow segmentation by temporal edge density information of adjacent frames. In: Electrical Engineering/Electronics, Computer, Telecommunications and Information Technology, ECTI-CON 2008. 5th International Conference on 1,pp.433-436, 2008

[9] Sun, Li, Wenjuam Sheng, and Yiqing Lui. Background modelling and its evaluation for complex scene. Multimedia tools and application 74.11, pp. 3947-3966, 2015.

[10] Xia, Haiying, Shuxiang Song, and Liping He. A modified Gaussian mixture background model via spatiotemporal distribution with shadow detection. Signal, Image and Video Processing 10.2, pp.343-350, 2016.

[11] Liu Wanjun, Li Lin. Moving objects detection algorithm of improved mixture Gaussian model based on entropy theory. Application Research of Computers, Vol.32, No.7, pp.2226-2233, 2015. 